\title{
Squeeze-Flow in the Presence of a Temperature Gradient: Effective Attraction between Asperites in the Friction Zone of Two Solids
}

\author{
Siegfried Hess, Ahmet Sow \\ Institute of Theoretical Physics, Technical University Berlin, Berlin, Germany \\ Communicated by D. Bedeaux, Leiden, The Netherlands
}

Registration Number 908

\begin{abstract}
The equations of hydrodynamics are solved for a slow squeeze-flow in the presence of a gradient of the viscosity which, in turn, is caused by a temperature gradient. The gradient induces an asymmetry of the spreading velocity and a drift of the center of mass towards the direction where the viscosity is decreasing. Due to this phenomenon, adjacent asperites in the friction zone between two solids sliding over each other experience an effective attraction.
\end{abstract}

\section{Introduction}

In dry friction between two solid materials, the actual contact occurs not on the whole surface, but in relatively small contact zones (asperites) which are of micrometer length scale [1], [2]. Due to the heat of friction, these asperites are hotter than the material around them. The temperature between two adjacent asperites is lower than on their outer sides. The temperature difference and the strong dependence of the viscosity on the temperature imply that the (plastic) squeeze-flow of the asperites under a load will be asymmetric, leading to an effective attraction between them. This is one mechanism which causes the observed agglomeration of asperites to larger hot spots in brakes [3], [4]. See Figure 1 for a schematic sketch of the geometry treated. Here an approximate solution of the relevant equations of hydrodynamics is presented with the aim of obtaining an estimate for the order of magnitude of the effects caused by a gradient of the viscosity, which in turn is due to a temperature gradient.

This article proceeds as follows. Firstly, in section 2, the equations of hydrodynamics, with a spatially dependent viscosity and the relevant boundary conditions for the squeeze-flow of a single asperite are stated. Then, in section 3, the solution of the squeeze-flow problem is presented for the simpler case of a spatially constant viscosity. Section 4 is devoted to the calculation of the squeeze-flow velocity in the presence of a 


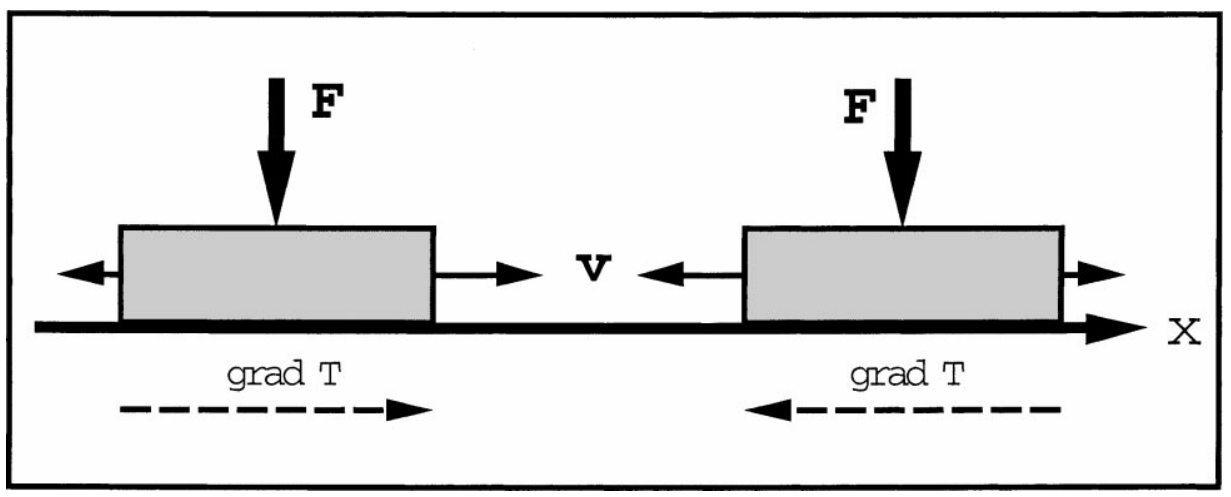

Fig. 1. Schematic squeeze-flow geometry in the $x y$-plane. The fast sliding motion is perpendicular to the plane of drawing (z-direction). The thick vertical arrows $\mathbf{F}$ indicate the normal force exerted on the top of the asperites. The horizontal arrows stand for the squeeze or spreading velocities $\mathbf{v}$ which are asymmetric due to the temperature gradients which are marked by the dashed arrows.

viscosity gradient which is assumed to be small enough such that it can be treated as a perturbation. The resulting drift velocity of the center of mass of an asperite and the effective force density associated with the gradient of the viscosity are given in section 5. In section 6 , the relation between the gradient of the viscosity and the temperature gradient is discussed and the order of magnitude of the temperature gradient outside an asperite, as well as that of the drift velocity are estimated.

\section{Hydrodynamics}

\subsection{Differential Equations}

The point of departure is the local conservation laws for mass and momentum, viz. the continuity equation $\partial \rho / \partial t+\nabla_{\nu}\left(\rho v_{\nu}\right)=0$, and

$$
\partial\left(\rho v_{\mu}\right) / \partial t+v_{\nu} \nabla_{\nu}\left(\rho v_{\mu}\right)+\nabla_{\mu} p+\nabla_{\nu} p_{\nu \mu}=0
$$

Here $\rho$ is mass density, $\mathbf{v}$ is the flow velocity, $p$ and $p_{\nu \mu}$ are the scalar (hydrostatic) pressure and components of the friction pressure. Greek subscripts indicate cartesian components, the summation convention is used for them.

In the following, we consider a slow creeping flow, i.e. time derivatives are set equal to zero and inertia terms are disregarded. Then the continuity equation reduces to $\rho=$ const and $\nabla_{\mu} v_{\mu}=0$. Furthermore, for the friction pressure Newton's ansatz $p_{\nu \mu}=-\eta\left(\nabla_{\nu} v_{\mu}+\nabla_{\mu} v_{\nu}\right)$ is used with the shear viscosity $\eta$. In the present study, the spatial dependence of the viscosity resulting from its dependence on the spatially varying temperature is taken into account. Insertion of this ansatz into the momentum balance equation leads, in the creeping flow limit, to the stationary Navier-Stokes equation

$$
\nabla_{\mu} p-\eta \Delta v_{\mu}-\left(\nabla_{\nu} \eta\right)\left(\nabla_{\nu} v_{\mu}+\nabla_{\mu} v_{\nu}\right)=0 .
$$


The bracket in $\left(\nabla_{\nu} \eta\right)$ indicates that only the viscosity is differentiated. Application of $\nabla_{\mu}$ on (2) yields

$$
\Delta p-2\left(\nabla_{\nu} \eta\right) \Delta v_{\nu}-\left(\nabla_{\nu} \nabla_{\mu} \eta\right)\left(\nabla_{\nu} v_{\mu}+\nabla_{\mu} v_{\nu}\right)=0
$$

This equation is used for determining the pressure.

\subsection{Special Geometry and Boundary Conditions}

Consider a long slab of a (highly) viscous substance on a flat surface. The height, width and length of the slab are denoted by $h, w=2 R$ and $L$, respectively. The limiting case $h \ll w \ll L$ is considered. This is typical for contact zones on a rotating disc brake [4]; the local curvature of the disc, however, is disregarded here. A pressure on the slab will lead to a squeeze flow, thus $h$ will decrease with the speed $u=-\dot{h}$. With $L$ assumed to be approximately constant, the width $w$ increases such that $h w \sim L^{-1}$ is approximately constant. To treat the squeeze flow in more detail, a coordinate system is introduced, where the $x$ and $y$ directions are along the width and the height of the slab, respectively, cf. Figure 1. In the application to be considered later, the (fast) motion which produces the heat of friction is in the $z$-direction, cf. Figure 1. For the moment, the sqeeze-flow velocity field has the components $v_{x}=v_{x}(x, y), v_{y}=v_{y}(x, y), v_{z}=0$. The pressure $p$ depends on $x$ and $y$, the viscosity is assumed to be a function of $x$. Then (2) and (3) simplify to

$$
\begin{array}{r}
\partial p / \partial x-\eta \Delta v_{x}-2 \eta^{\prime} \partial v_{x} / \partial x=0 \\
\partial p / \partial y-\eta \Delta v_{y}-\eta^{\prime} \partial v_{x} / \partial y=0
\end{array}
$$

and

$$
\Delta p-2 \eta^{\prime} \Delta v_{x}-2 \eta^{\prime \prime} \partial v_{x} / \partial x=0
$$

where $\eta^{\prime}=\partial \eta / \partial x, \eta^{\prime \prime}=\partial^{2} \eta / \partial x^{2}$, and $\Delta=\partial^{2} / \partial x^{2}+\partial^{2} / \partial y^{2}$ stands for the twodimensional Laplacian.

With the origin of the coordinate system on the lower side in the middle of the slab, the boundary conditions are

$$
v_{x}(x, 0)=v_{x}(x, h)=0, \quad v_{y}(x, 0)=0, \quad v_{y}(x, h)=\dot{h}=-u
$$

Furthermore, it is assumed that the pressure on the top, $y=h$, is exerted by a solid material which can take up a spatial variation of the pressure in the $x$ direction. The normal force (load) $F_{n o r m}$ acting on the slab is given by $F_{n o r m} / L=\int_{-R}^{R}\left(p(x, h)-p_{0}\right) d x$, where $p_{0}$ is the constant pressure outside the $x$-interval $[-R, R]$. In Figure 1 this force is denoted by $\mathbf{F}=-F_{\text {norm }} \mathbf{e}_{y}$, where $\mathbf{e}_{y}$ is a unit vector parallel to the $y$ direction.

Later, the case $v_{z}=v_{z}(y)$, as encountered in plane Couette flow in the $z$ direction, will be considered. Apart from end corrections and for the case where the motion in the $x$ direction is slow and the motion in the $y$ direction is even slower, the squeeze flow and the Couette flow can be treated as practically decoupled processes. 


\section{Squeeze-Flow for Constant Viscosity}

When the viscosity $\eta$ is independent of the spatial coordinates, the solution of the differential equations (4), (3) with the boundary conditions stated above is, for $-R \leq x \leq R$ and $0 \leq y \leq h$,

$$
v_{x}=6 u \frac{x}{h} \frac{y}{h}\left(1-\frac{y}{h}\right), \quad v_{y}=-u \frac{y^{2}}{h^{2}}\left(3-2 \frac{y}{h}\right)
$$

and

$$
p-p_{0}=6 \eta \frac{u}{h}\left(\frac{R^{2}-x^{2}}{h^{2}}-\frac{y}{h}\left(1-\frac{y}{h}\right)\right) .
$$

Notice that $v_{y}$ is independent of $x$, as it should be for this case. For a sketch of the velocity field see the upper graph of Figure 2.

The squeeze velocity $v_{x}$, averaged over the height, is $v_{s q}=u x / h$. For $x=R$ one has $v_{s q}=\dot{R}=u R / h=-R \dot{h} / h$ such that $R h=$ const is recovered. The strong enhancement of the horizontal squeeze velocity by the factor $R / h$, for $R \gg h$, over the vertical speed $u$ is the essence of the "squeeze" phenomenon.

The normal force per length is given by

$$
F_{\text {norm }} / L=4 u \eta R^{3} / h^{3} .
$$

As a side remark, the corresponding squeeze force for a circular disc with radius $R$ [5], [6] is $F_{\text {norm }}=3 \pi u \eta R^{4} / h^{3}$. The average normal pressure which has to be exerted on the slab in order to achieve the velocity $u$ of the top layer is

$$
\sigma=F_{\text {norm }} /(2 R L)=2 u \eta R^{2} / h^{3} .
$$

For comparison, the corresponding quantity for a circular disc with radius $R$ is $\sigma=F_{\text {norm }} /\left(\pi R^{2}\right)=3 u \eta R^{2} / h^{3}$.

Since $u=-\dot{h}$, these relations can be used to determine $h=h(t)$ and $R=R(t)$, with $R h=R_{0} h_{0}=$ const, either for a constant force or a constant pressure. The resulting solution, for the constant pressure, is $\left(h_{0} / h(t)\right)^{4}-1=\left(R(t) / R_{0}\right)^{4}-1=$ $2(\sigma / \eta)\left(h_{0} / R_{0}\right)^{2} t$. The characteristic time $t_{c}$, where $h$ has decreased by a factor $1 / 2$ and $R$ has increased by a factor 2 , is $t_{c}=(15 / 2)(\eta / \sigma)\left(R_{0} / h_{0}\right)^{2}$. For $\sigma=10^{9} \mathrm{~Pa}$, the penetration hardness, i.e. the pressure where a metal like iron starts to flow under a load, $R_{0} / h_{0}=10^{2}$ and $\eta=10^{4} \mathrm{Pas}$, this time is $0.75 \mathrm{~s}$.

When the force is kept constant, similar relations hold true, in particular one has $\left.\left(h_{0} / h\right)(t)\right)^{5}-1=\left(R(t) / R_{0}\right)^{5}-1=(3 / 2 \eta)\left(F_{\text {norm }} / L\right)\left(h_{0}^{2} / R_{0}^{3}\right) t$, and $t_{c}=(32 / 3)\left(\eta L R_{0} / F_{\text {norm }}\right)\left(R_{0} / h_{0}\right)^{2}$.

With the initial pressure $F_{\text {norm }} /\left(L R_{0}\right)$ put equal to $10^{9} \mathrm{~Pa}$ and the other quantities just as above, one now finds the slightly larger time $t_{c} \approx 1.1 \mathrm{~s}$. For $R_{0} / h_{0}=10$, rather than the value used above, these characteristic times are shorter by a factor $10^{-2}$. For 
order of magnitude considerations one may introduce a characteristic 'spreading' time $t_{s p r}$ by dividing the distance $R / 2$ by the squeeze velocity $v_{s q}(x)=u x / h$ at $x=R$. Thus,

$$
t_{s p r}=\frac{1}{2} \frac{R}{v_{s q}(R)}=\frac{1}{2} \frac{h}{u}=\frac{\eta}{\sigma}\left(\frac{R}{h}\right)^{2} .
$$

The spreading due to the squeeze-flow over distances comparable with their width is considerably faster for narrower asperites. For a spatially constant viscosity, the spreading is symmetric in the $+x$ and $-x$ directions. This symmetry is broken by a gradient of the viscosity.

\section{Squeeze-Flow for a Spatially Varying Viscosity}

Next the case of a spatially varying viscosity of the form

$$
\eta=\eta_{0}+\eta^{\prime} x
$$

is considered, where $\eta_{0}$ and the derivative $\eta^{\prime}=\partial \eta / \partial x$ are constant. The effect of the spatial variation is treated as a perturbation. This means the ratio $\epsilon=\eta^{\prime} R / \eta_{0}$ is assumed to be small compared with 1 . Thus we write the velocity as $\mathbf{v}=\mathbf{v}^{(1)}+\mathbf{v}^{(2)}$, with $\mathbf{v}^{(1)}$ given by the solution (7) for a spatially constant viscosity, and we seek a solution for the distortion $\mathbf{v}^{(2)}$ which is linear in the perturbation parameter $\epsilon$. Similarly, we decompose the pressure $p=p^{(1)}+p^{(2)}$ with $p^{(1)}$ given by (8), where $\eta$ is replaced by $\eta_{0}$. Then the equation for $v_{x}^{(2)}$, as inferred from (4), is

$$
\partial p^{(2)} / \partial x-\eta_{0} \Delta v_{x}^{(2)}-\eta^{\prime} x \Delta v_{x}^{(1)}-2 \eta^{\prime} \partial v_{x}^{(1)} / \partial x=0 .
$$

The equation for $p^{(2)}$ following from (5) is, in order $\epsilon$,

$$
\Delta p^{(2)}=2 \eta^{\prime} \Delta v_{x}^{(1)}=-24 u \eta^{\prime} x / h^{3} .
$$

Thus, one obtains

$$
p^{(2)}=6 u \eta^{\prime} \frac{x}{h}\left(\frac{\alpha}{3 h^{2}}\left(R^{2}-x^{2}\right)+(2-\alpha) \frac{y}{h}\left(1-\frac{y}{h}\right)\right),
$$

where the constant $\alpha$, which is associated with the solution of the homogeneous equation, is determined later. The "boundary condition" $p^{(2)}( \pm R, h)=0$ has been used. Insertion of the expression (14) for $p^{(2)}$ and of (7) for the velocity $v_{x}^{(1)}$ into (12) yields

$$
\Delta v_{x}^{(2)}=6 \frac{u}{h} \frac{\eta^{\prime}}{\eta_{0}}\left(\frac{\alpha}{3}\left(\frac{R}{h}\right)^{2}+(2-\alpha)\left(\frac{x}{h}\right)^{2}-\alpha \frac{y}{h}\left(1-\frac{y}{h}\right)\right) .
$$

Next, the ansatz $v_{x}^{(2)}=-3(u / h)\left(\eta^{\prime} / \eta_{0}\right)\left(A+B x^{2} / h^{2}\right)(y / h)(1-y / h)$ is made for the distortion of the velocity which obeys the boundary conditions at $y=0$ and $y=h$. 
The constants $A$ and $B$ have to be determined. Application of the Laplacian on this ansatz yields an expression proportional to $\left(A R^{2}+B x^{2}\right) / h^{2}-B(y / h)(1-y / h)$. Compatibility with (15) implies $A=\alpha / 3, B=2-\alpha$ and $B=\alpha$. The second and third of these relations lead to $\alpha=1$ and $B=1$, the first one then yields $A=1 / 3$. Thus, the solution for the $x$ component of the gradient-induced velocity is

$$
v_{x}^{(2)}=-3 u \frac{\eta^{\prime} h}{\eta_{0}}\left(\frac{1}{3}\left(\frac{R}{h}\right)^{2}+\left(\frac{x}{h}\right)^{2}\right) \frac{y}{h}\left(1-\frac{y}{h}\right) .
$$

Averaged over the height of the slab, this expression implies

$$
v_{s q}^{(2)}=-\frac{1}{2} u \frac{\eta^{\prime} h}{\eta_{0}}\left(\frac{1}{3}\left(\frac{R}{h}\right)^{2}+\left(\frac{x}{h}\right)^{2}\right)
$$

for the contribution to the squeeze velocity associated with the gradient of the viscosity. Thus, at $x=R$, the total squeeze velocity is

$$
v_{s q}=v_{s q}^{(1)}+v_{s q}^{(2)}=\left(1-\frac{2}{3} \frac{\eta^{\prime} R}{\eta_{0}}\right) u \frac{R}{h} .
$$

For $\eta^{\prime}<0$ the squeeze velocity will increase at $x=R$ and decrease at $x=-R$ according to the relative decrease of the viscosity over the length $R$. The characteristic time $t_{s p r}^{+}$for spreading in the $x$ direction is shorter than the time for spreading the same distance in the $-x$ direction. In particular, one has

$$
t_{s p r}^{+}=\frac{1}{2} \frac{R}{v_{s q}(R)}=\left(1-\frac{2}{3} \frac{\eta^{\prime} R}{\eta_{0}}\right)^{-1} \frac{\eta}{\sigma}\left(\frac{R}{h}\right)^{2} .
$$

A word of caution is in order. The approximate solution obtained here from the stationary version of the equations of hydrodynamics is only valid for the boundary conditions used, applicable for a short time. On longer time scales, the boundary conditions have to be modified due to the shift of the center of mass towards the side where the viscosity is smaller. Furthermore, the upper side of the slab is tilted under the pressure. From the continuity equation one infers $\partial v_{y}^{(2)} / \partial y=-\partial v_{x}^{(2)} / \partial x$. Insertion of (16) into this relation shows, that $v_{y}^{(2)}$, in contradistinction to $v_{y}^{(1)}$, does depend on $x$. The resulting velocity, $v_{y}^{(2)}=\frac{1}{2} u \frac{\eta^{\prime} R}{\eta_{0}} \frac{x}{R}\left(\frac{y}{h}\right)^{2}\left(3-2 \frac{y}{h}\right)=-\frac{1}{2} \frac{\eta^{\prime} R}{\eta_{0}} \frac{x}{R} v_{y}^{(1)}$, vanishes at $y=0$, as it should. For $y=h$, however, this velocity depends on $x$, cf. the lower graph of Figure 2.

Such a motion can be caused by a rigid body on top of the viscous slab when, in addition to the speed $u$ in the $y$ direction, it performs a rotation about an axis parallel to the $z$ direction with the angular velocity $\omega_{z}=(1 / 2) u \eta^{\prime} / \eta_{0}$. This more general case, however, is not studied here. We are interested in an estimate of the order of magnitude of the effect caused by a spatially varying viscosity rather than a full hydrodynamic solution. 


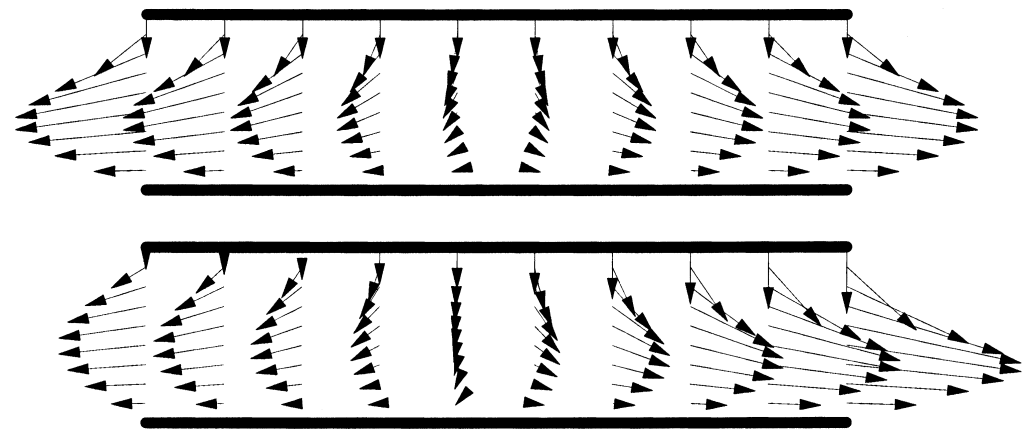

Fig. 2. Velocity fields for a constant viscosity (upper graph) and for the viscosity decreasing towards the right (lower graph) for $\eta^{\prime} h / \eta_{0}=0.25$.

\section{Drift Velocity and Effective Force}

In contradistinction to the case of a constant viscosity, the center of mass of an asperite experiences a drift when the viscosity is spatially inhomogeneous. An average of the velocity as given by (17) over $x$, from $-R$ to $R$, yields the center of mass drift velocity

$$
v_{d r i f t}=-\frac{1}{3} u \frac{\eta^{\prime} R}{\eta_{0}} \frac{R}{h} .
$$

On the other hand, a force density $\mathscr{F}_{x}$ leads to a drift in the $x$ direction equal to $\mathscr{F}_{x} h^{2} /\left(12 \eta_{0}\right)$ when the fluid is confined between flat plates seperated by the distance $h$. Thus the drift caused by the gradient of the viscosity is equal to that due to an effective force density given by

$$
\mathscr{F}_{x}=-4 u \frac{R^{2}}{h^{3}} \frac{\partial \eta}{\partial x}=-2 \frac{\eta^{\prime}}{\eta_{0}} \sigma,
$$

where $\sigma$ is the average normal pressure cf. (10). An estimate for the order of magnitude of the relative spatial variation of the viscosity is presented next.

\section{Effective Attraction due to a Temperature Gradient}

\subsection{Spatial Variation of the Viscosity}

As mentioned before, the spatial variation of the viscosity is considered to be caused by a spatially varying temperature $T$. Thus, one has $\epsilon=\left(\eta_{0}\right)^{-1} \eta^{\prime} R=\left(\eta_{0}\right)^{-1}(d \eta / d T) T^{\prime} R$ for the quantity determining the magnitude of the effect of a spatial gradient on the sqeeze velocity, cf. (18). Upon the assumption that the temperature dependence of the viscosity is of Arrhenius type, viz. $\eta \sim \exp \left(T_{A} / T\right)$ with a constant Arrhenius temperature $T_{A}$, one obtains $\epsilon=-T_{A} \delta T / T^{2}$, where $\delta T=T^{\prime} R$ is the (linear) variation of $T$ over the distance $R$. Typically, $T_{A}$ is larger than the melting temperature, e.g. by 
a factor of 2. Then, for temperatures in the vicinity of the melting temperature, $\delta T / T \approx 0.5$ implies $\epsilon \approx 1$. Of course, the linear perturbation theory presented above is no longer valid when $\epsilon$ is so large. On the other hand, these considerations show that the effects discussed here are of observable magnitude.

\subsection{Temperature Profile between two Asperites}

Now two asperites are considered which are both elongated in the $z$ direction and have the (initial) $R_{8}$ (Fig. 1). They are assumed to be relatively close to each other in the $x$ direction. Let the center of mass of asperite " 1 " be located at $x=0$, that of " 2 " at $x=R_{2}>2 R$. The temperature gradient $T^{\prime}=\partial T / \partial x$, which one asperite feels due to the heat friction generated by its (lateral) neighbor, is estimated as follows. First, the temperature of the support carrying the slab " 1 " undergoing a viscous flow of plane Couette type in the $z$ direction is determined from the stationary heat conduction equation $-\lambda \Delta T=\dot{Q}$. Here

$$
\dot{Q}_{v i s c}=\eta\left(\partial v_{z} / \partial y\right)^{2}=\eta\left(\frac{v_{z}(h)}{h}\right)^{2}
$$

is the heat produced by the fast viscous flow in the $z$ direction. The coefficient $\lambda$ is the (effective) heat conductivity. The viscous heat caused by the slow squeeze flow is disregarded. Alternatively, the heat generated by "dry" friction characterized by the friction coefficient $\mu$, divided by the volume $h 2 R L$ of an asperite is $F_{z}^{f r i c} v_{z} /(h 2 R L)$, where it is understood that $v_{z}$ is the (constant) velocity in the $z$ direction. The friction force $F_{z}^{\text {fric }}$ is related to the normal force $F_{n o r m}=\sigma 2 R L$ by $F_{z}^{\text {fric }}=\mu F_{\text {norm }}$. Thus, one obtains

$$
\dot{Q}_{\text {fric }}=\mu \sigma \frac{v_{z}}{h}
$$

for the energy density produced by dry friction.

With the temperature at $x=0$, denoted by $T_{m}$, the solution of the heat conduction equation is $T=T_{m}-(1 / 2) \dot{Q} x^{2} / \lambda$, for $|x| \leq R$, and $T=T_{m}+(1 / 2) \dot{Q} R^{2} / \lambda-\dot{Q} R x / \lambda$, for $|x| \geq R$. Thus, one has $T^{\prime}=-\dot{Q} R / \lambda$ on the right hand side of the asperite located at $x=0$. This is the temperature gradient which asperite " 2 " feels. Similarly, the heat generated by asperite " 2 " causes a temperature gradient $T^{\prime}=\dot{Q} R / \lambda$ on its left hand side which is felt by asperite " 1 ".

A quick estimate shows that a temperature difference $\delta T=T^{\prime} R$ of $1000 \mathrm{~K}$ can easily be reached, e.g. for $R=10^{-4} \mathrm{~m}$ and $\lambda=10^{2} \mathrm{Wm}^{-1} \mathrm{~K}^{-1}$ (a value typical for a metal like iron) when one uses $v_{z} / h=10^{3} s^{-1}, \eta=10^{3} \mathrm{Pas}$ in the case of viscous heating and $v_{z} / h=10 s^{-1}, \mu=0.1, \sigma=10^{9} \mathrm{~Pa}$ for the case of dry friction. Of course, larger velocities are possible which seem to imply still larger temperature differences. Other loss mechanisms for the heat generated, however, in addition to the heat conduction considered here, have to be taken into account. Alternatively, $\lambda$ has to be interpreted as a considerably larger effective transport coefficient. 


\subsection{Drift Velocity}

Combining the results presented above, we finally obtain for the drift velocity, cf. (20)

$$
v_{d r i f t}=-\frac{1}{3} \frac{\sigma}{\eta_{0}} h^{2} \frac{T d \eta}{\eta_{0} d T} T^{\prime}=-\frac{1}{3} \frac{\sigma}{\eta_{0}} h^{2} \frac{T d \eta}{\eta_{0} d T} \frac{\dot{Q} R}{T \lambda}
$$

where $\sigma$ is recalled as the (average) normal pressure. With the heat generated given by either (22) or (23), the corresponding expressions for the drift velocity are

$$
v_{d r i f t}^{v i s c}=-\frac{1}{3} \sigma \frac{T d \eta}{\eta_{0} d T} v_{z}^{2} \frac{R}{T \lambda}
$$

and

$$
v_{d r i f t}^{f r i c}=-\frac{1}{3} \frac{\sigma}{\eta_{0}} h \frac{T d \eta}{\eta_{0} d T} \mu \sigma v_{z} \frac{R}{T \lambda} .
$$

With the realistic estimate $-(1 / 3) T d \eta /(\eta d T) \approx 1$, the drift velocity in the lateral direction may easily reach one tenth of the longitudinal velocity $v_{z}$, in both cases. The direction of the drift is towards the next laterally adjacent asperite. Thus, this drift acts like an attractive force between the asperites. The relation

$$
\mathscr{F}_{x}=12 \eta_{0} v_{\text {drift }} / h^{2}
$$

can be used to infer expressions for the force density corresponding to the cases described by (25) and (26). Notice that the force density given here depends on the size of the asperites but not on their distance of seperation. The later fact is due to the idealization of very long asperites where the temperature gradient outside the heat source is constant. In a more realistic situation, the temperature gradient and consequently the drift velocity and the effective force will be smaller for larger seperations between asperites. For a circular heat source e.g., the outside temperature gradient decreases inversely proportional to the distance.

\section{Concluding Remarks}

In this article, the influence of a gradient of the viscosity, caused by a temperature gradient, on the spreading velocity in a squeeze-flow has been calculated in first order in the gradients and for a simple geometry. The resulting gradient-induced drift of the center of mass of an asperite is of significant magnitude. Furthermore, an effective force was given which implies an attraction between adjacent asperites and eventually leads to an agglomeration of small asperites to larger hot spots. The dynamics of the agglomeration process, however, is outside the scope of the present study.

The analysis presented is based on the Newtonian relation between the friction pressure tensor and the gradient of the flow velocity (deformation rate tensor). Of course, the squeeze flow problem can and has been treated [7] for fluids with a more 
complex rheological behavior. In an application to sliding friction as encountered in brakes, the shear viscosity used here has to be looked upon as an effective transport coeffcient for the slow squeeze flow of a material in a state driven far from equilibrium by the fast sliding motion. Viscous and plastic flow processes modify the local structure [8], [9], [10] and affect the transport coefficients [11]. The measurement and calculation of transport coefficients for systems far from equilibrium was beyond the scope of the present study and it remains to be a challenge.

\section{Acknowledgements}

This work has been performed under the auspices of the Sonderforschungsbereich SFB 605 "Elementarreibereignisse" of the Deutsche Forschungsgemeinschaft (DFG). Financial support by the DFG is gratefully acknowledged.

\section{References}

[1] Bowden, F. P., Tabor, D., Friction and Lubrication, Methuen, London, 1967.

[2] Persson, B. N. J., Sliding Friction, Springer, Berlin 1998.

[3] Anderson, A. E., Knapp, R. A., Hot spotting in automotive friction systems, Wear 135 (1990), 319-337.

[4] Severin, D., Musiol, F., Der Reibprozess in trockenlaufenden mechanischen Bremsen und Kupplungen, Konstruktion, 47 (1995), 59-68.

[5] Stefan, J., Sitzber. K. Akad. Wiss. Math. Natur., Wien, 69, 2, (1874), 713-735, as quoted by Bird, R. B., Armstrong, R. C., and Hassager, O., Dynamics of Polymeric Liquids, Vol. 1: Fluid Mechanics, p. 19, Wiley, New York, 1977.

[6] Landau, L. D., Lifschitz, E. M., Hydrodynamik, p. 79, Akademie-Verlag, Berlin, 1966.

[7] Sherwood, J. D., Durban, D., Squeeze-flow of a Herschel-Bulkley fluid, J. Non-Newtonian Fluid Mech., 77 (1998), 41-57.

[8] Hess, S., Structure of liquids and solids in nonequilibrium, J. de Physique, 46 (1985), 191-209.

[9] Hess, S., Flow properties and structure of anisotropic fluids studied by Non-Equilibrium Molecular Dynamics, and flow properties of other complex fluids: Polymeric liquids, ferro-fluids and magneto-rheological fluids, in: Advances in the Computer Simulations of Liquid Crystals, Eds. O. Pasini and C. Zannoni, p. 189-233, Kluwer, Dordrecht, Netherlands, 2000.

[10] Pyzalla, A., Stress and strain measurements: X-rays and neutrons, Physica B, 276-278 (2000), 833-836.

[11] Hess, S., Simple and complex fluids under shear, in: Monte Carlo and Molecular Dynamics of Condensed Matter Systems, Eds. K. Binder and G. Ciccotti, p. 825-841, IPS Conf. Proc. 49, Bologna, 1996.

Paper received: 2001-04-19

Paper accepted: 2001-10-15

Prof. Dr. S. Hess

Dipl. Phys. A. Sow

Institute of Theoretical Physics

Technical University Berlin, PN 7-1

Hardenbergstr. 36

10623 Berlin

Germany

E-mail: s.hess@physik.tu-berlin.de 